\title{
THE VVV SURVEY REVEALS CLASSICAL CEPHEIDS TRACING A YOUNG AND THIN STELLAR DISK ACROSS THE GALAXY'S BULGE
}

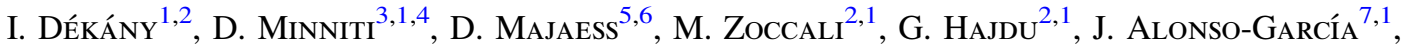 \\ M. Catelan ${ }^{2,1}$, W. Gieren ${ }^{8,1}$, and J. Borissova ${ }^{9,1}$ \\ ${ }_{1}^{1}$ Instituto Milenio de Astrofísica, Santiago, Chile; idekany@astro.puc.cl \\ ${ }^{2}$ Instituto de Astrofísica, Facultad de Física, Pontificia Universidad Católica de Chile, Av. Vicuña Mackenna 4860, Santiago, Chile \\ ${ }^{3}$ Departamento de Física, Facultad de Ciencias Exactas, Universidad Andres Bello, República 220, Santiago, Chile \\ ${ }_{5}^{4}$ Vatican Observatory, V00120 Vatican City State, Italy \\ ${ }^{5}$ Saint Mary's University, Halifax, Nova Scotia, Canada \\ ${ }^{6}$ Mount Saint Vincent University, Halifax, Nova Scotia, Canada \\ ${ }^{7}$ Unidad de Astronomía, Fac. Cs. Básicas, Universidad de Antofagasta, Avda. U. de Antofagasta 02800, Antofagasta, Chile \\ ${ }^{8}$ Departamento de Astronomía, Universidad de Concepción, Casilla 160-C, Concepción, Chile \\ ${ }^{9}$ Instituto de Física y Astronomía, Universidad de Valparaíso, Av. Gran Bretaña 1111, Valparaso, Chile \\ Received 2015 July 28; accepted 2015 September 26; published 2015 October 16
}

\begin{abstract}
Solid insight into the physics of the inner Milky Way is key to understanding our Galaxy's evolution, but extreme dust obscuration has historically hindered efforts to map the area along the Galactic mid-plane. New comprehensive near-infrared time-series photometry from the VVV Survey has revealed 35 classical Cepheids, tracing a previously unobserved component of the inner Galaxy, namely a ubiquitous inner thin disk of young stars along the Galactic mid-plane, traversing across the bulge. The discovered period (age) spread of these classical Cepheids implies a continuous supply of newly formed stars in the central region of the Galaxy over the last 100 million years.
\end{abstract}

Key words: Galaxy: bulge - Galaxy: disk - Galaxy: general - Galaxy: stellar content - stars: variables: Cepheids Supporting material: data behind figure

\section{INTRODUCTION}

The inner Milky Way is dominated by a peanut-shaped bulge (McWilliam \& Zoccali 2010; Wegg \& Gerhard 2013) that flares up from a prominent Galactic bar (Nakada et al. 1991), and its structural and kinematical properties are consistent with a formation scenario driven by the instabilities of a multicomponent stellar disk (Ness et al. 2013; Gardner et al. 2014; Ness et al. 2014; Zoccali et al. 2014; Di Matteo et al. 2015). The majority of its constituent stars are very ( $\gtrsim 8$ billion years) old (Ortolani et al. 1995; Zoccali et al. 2003; Brown et al. 2010), implying that its formation occurred at early epochs of the Milky Way's evolution. Although intermediateage stars are also present in the bulge (van Loon et al. 2003; Bensby et al. 2013), their origin, nature, and ubiquity are poorly understood, owing partly to sizable age uncertainties and possible biases from small sample sizes and contamination by foreground disk stars. Yet it is well known that the innermost core $(R \lesssim 300 \mathrm{pc})$ of the Galaxy hosts stars with ages ranging from a few million to several billion years (e.g., Serabyn \& Morris 1996; van Loon et al. 2003; Matsunaga et al. 2011). This "nuclear bulge" has rather distinct physical properties from the rest of the bulge and is related to the Central Molecular Zone, but neither its transition to the surrounding bulge regions nor the triggering process for star formation and its history are well understood (see, e.g., Launhardt et al. 2002).

Our global picture of the inner Galaxy is linked primarily to observations of the outer bulge, i.e., at Galactic latitudes higher than $\sim 2^{\circ}$. That deficit arises from extreme obscuration by interstellar dust, high source density, and confusion with foreground disk populations. The properties of the bulge region at low latitudes have thus remained largely unexplored, leaving ambiguities concerning the interplay of the nuclear bulge and the various components of the boxy bulge and surrounding outer stellar disk. The VISTA Variables in the Vía Láctea (VVV) ESO Public Survey (Minniti et al. 2010) presents a means to ameliorate the situation by opening the time-domain in the near-infrared. That is particularly important as classes of variable stars, such as classical Cepheids, are indicators of young stellar populations (e.g., Catelan \& Smith 2015), and can yield critical insight as demonstrated here.

\section{DISCOVERY AND CHARACTERIZATION OF THE CEPHEIDS}

We performed a comprehensive near-infrared variability search using VVV Survey data collected between 2010 and 2014 , in a $\sim 66$ square degree area in the central bulge $\left(-10.5 \lesssim l \lesssim+10^{\circ},-1.7 \lesssim b \lesssim+2^{\circ}\right.$, aligned with VVV image borders). We excluded two fields lying toward the nuclear bulge owing to extreme source crowding. Time-series photometry for $\sim 10^{8}$ point sources were analyzed, with up to $70 K_{s}$-band measurements per object, together with color information from 1-5 independent measurements in the $Z, Y, J$, and $H$ bands. The data processing, calibration, and light curve analysis were conducted in the same way as in our previous study on the Twin Cepheids beyond the bulge (Dékány et al. 2015), and are based on standard VVV Survey data products (Irwin et al. 2004; Minniti et al. 2010; Catelan et al. 2013). We detected a sample of approximately $3 \cdot 10^{5}$ objects which displayed putative light variations, and scanned the results for Cepheids possessing pulsation periods in the range of 4-50 days. The lower limit mitigates confusion between the pulsation modes (e.g., Macri et al. 2015), while the upper limit was constrained by the sampling of the photometric time- 

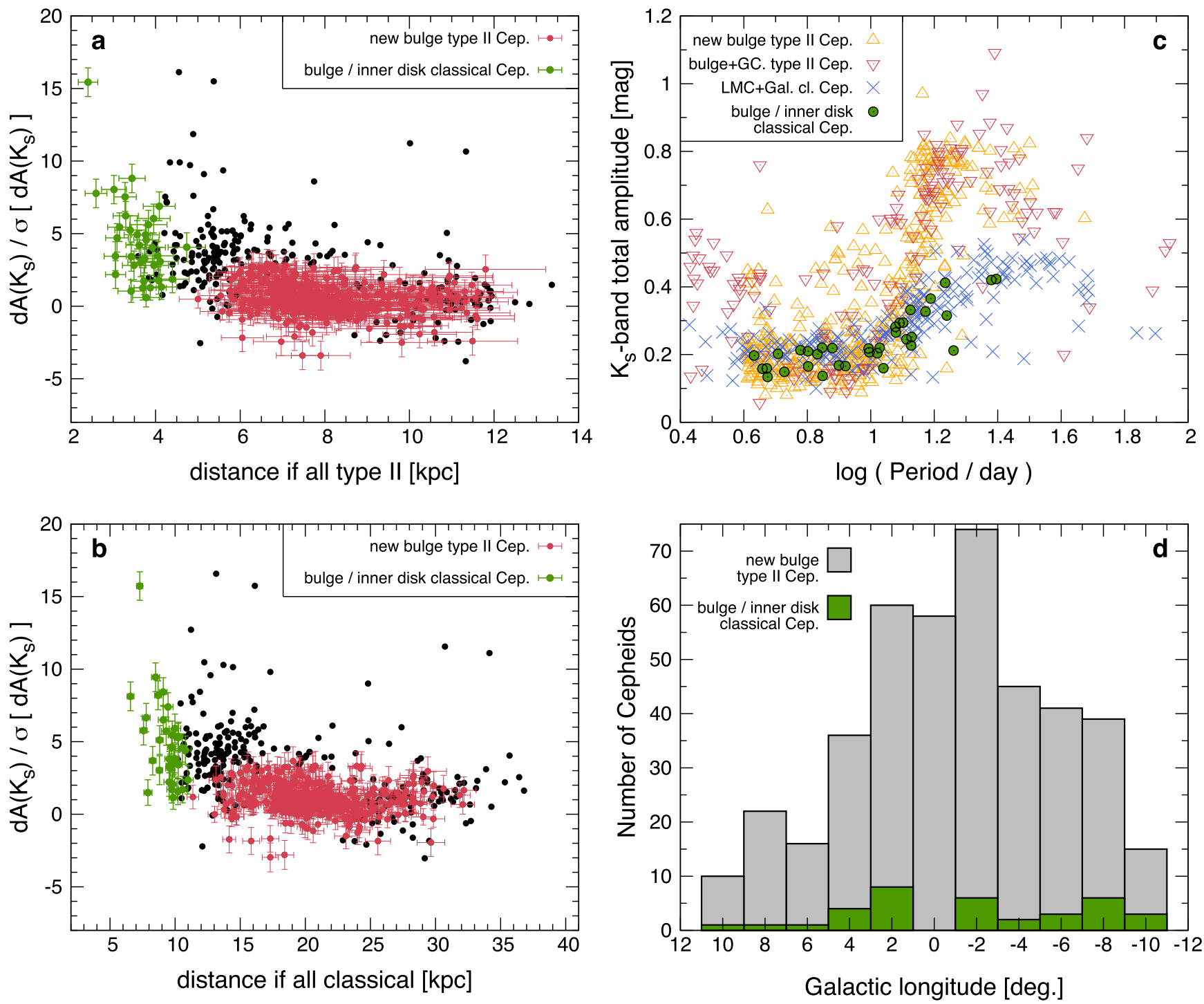

Figure 1. Selection of classical Cepheids. Panel (a): under the assumption that all Cepheids in our sample are of type II, this figure shows the deviation of their computed extinction relative to the VVV reddening map (Gonzalez et al. 2012) divided by the uncertainty, as a function of the distance. Error bars show $1 \sigma$ uncertainty ranges. Green points: the 35 classical Cepheids inside the bulge volume; red points: new type II bulge Cepheids with solid classifications; black points: rest of the sample. Black points at short distances in this figure represent candidate classical Cepheids beyond the bulge, while those at larger distances are type II Cepheid candidates outside the bulge or objects with uncertain classification. Panel (b): as panel (a), but showing values computed under the assumption that all Cepheids are of the classical type. Panel (c): $K_{s}$ total amplitude vs. $\log P$ diagram of classical Cepheids from the LMC (Persson et al. 2004) and the Galactic field (Monson \& Pierce 2011; blue crosses), previously known type II Cepheids in the bulge (Soszyński et al. 2011; $K_{s}$ amplitudes derived from VVV data) and in Galactic globular clusters (Matsunaga et al. 2006; red triangles), and new type II Cepheids from our study (orange triangles). Green points show the new classical Cepheids in the inner Galaxy. Panel (d): histograms of the discovered classical and type II Cepheid sample as a function of Galactic longitude.

series. We found 655 fundamental-mode Cepheid candidates based on their periods, amplitudes, and asymmetric light curve shapes. A large fraction of these objects were only detected in the $H$ and $K_{s}$ bands given the extreme reddening.

For computing the distances and extinctions of the Cepheids, we employed the period-luminosity (PL) relations (a.k.a. Leavitt Law; Matsunaga et al. 2009; Monson et al. 2012) adopted in our previous study (Dékány et al. 2015), adjusted to match the latest and most accurate distance modulus for the Large Magellanic Cloud (LMC; Pietrzyński et al. 2013). The color excess was converted to an extinction using a selectiveto-absolute extinction ratio found toward the Galactic Center (GC; Nishiyama et al. 2009), once converted into the VISTA photometric system (see Dékány et al. 2015). For each object, a distance and extinction were computed under both the assumptions that the target is a classical and a type II Cepheid. We subsequently identified the correct solution and class upon further analysis.

Following the approach adopted in our previous study (Dékány et al. 2015), uncertainties tied to the distance and extinction were estimated by Monte Carlo simulations. The distances exhibit $1 \%-3 \%$ precision and $8 \%-10 \%$ accuracy. The latter is dominated by the uncertainty in the adopted extinction curve. The systematic uncertainty might be larger, possibly up to $\sim 20 \%$, if variations in the extinction's wavelength dependence, either as a function of Galactic longitude or along the sight-line, significantly exceed those found previously toward the inner bulge (Nishiyama et al. 2009).

Type II Cepheids, i.e., old, low-mass, He-shell burning pulsating stars in the classical instability strip (Catelan \& Smith 


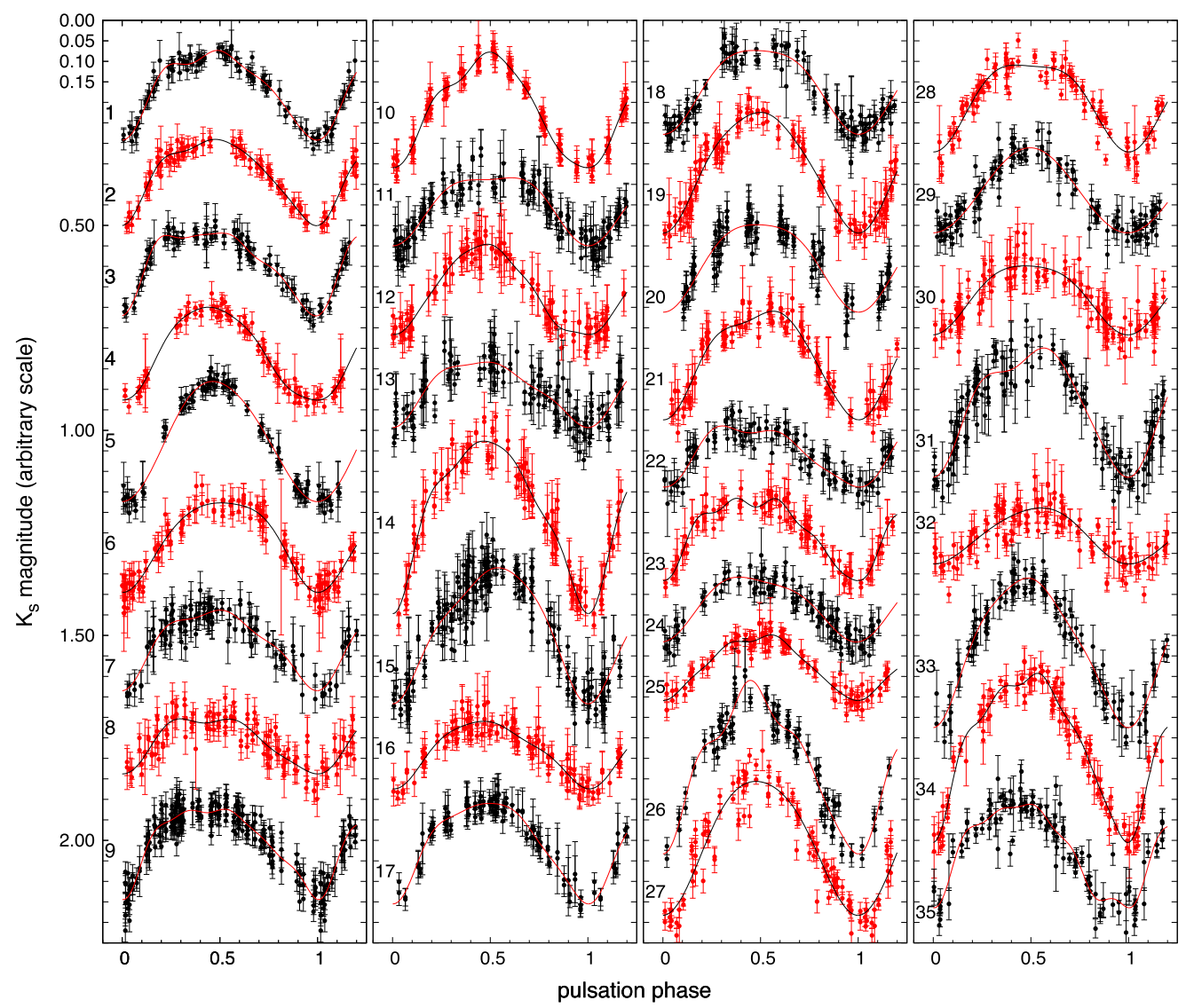

Figure 2. $K_{s}$-band phase diagrams of the classical Cepheids in the inner Galaxy (on arbitrary magnitude scale). Curves denote Fourier series fits to the data, identifiers are shown on the left. The data used to create this figure are available.

2015), populate the same range of periods as classical Cepheids. The two Cepheid classes share similar near-infrared light curves (see, e.g., Matsunaga et al. 2011; Catelan \& Smith 2015; Dékány et al. 2015). Since the bulge contains a sizable population of type II Cepheids with a centrally concentrated distribution (Soszyński et al. 2011), our sample is expected to be dominated by these objects. Therefore, additional observational information is required to identify classical Cepheids in our sample. The critical information is conveyed by an extinction map of the bulge, based on the analysis of its red clump stars (Gonzalez et al. 2012).

Classical and type II Cepheids have similar intrinsic colors but rather different luminosities (Matsunaga et al. 2009; Monson et al. 2012), and consequently their PL relations yield similar extinctions but very different distances when applied to the same object. A Cepheid's type can be determined if its distance and extinction are inconsistent with the cumulative extinction up to the bulge in its direction, computed under one of the two possible assumptions for its type (Matsunaga et al. 2011; Dékány et al. 2015). Figures 1(a)-(b) show the difference between the extinction values predicted by the PL relations and the bulge extinction map, normalized by its uncertainty as a function of distance, when extinction and distance are computed under the assumption that all detected Cepheids are either classical or type II. There are several Cepheids in our sample for which the computed extinction was within $3 \sigma$ agreement with, or higher than the predicted cumulative extinction up to the bulge toward their sight-lines, but for which the type II assumption yields short distances
(3-4 kpc), making them bona fide classical Cepheid candidates. While most of them reside in the disk at the far side of the bulge as expected, we found 35 stars (green points in Figure 1) that are likewise located within the bulge volume (see, e.g., Wegg \& Gerhard 2013).

These 35 stars could be type II Cepheids only if they were located by chance beyond thick dust clouds of small angular sizes $\left(<1^{\prime}\right)$, otherwise the $2^{\prime} \times 2^{\prime}$ resolution of the VVV reddening map would be sensitive to those. To investigate this unlikely possibility, we examined the color images and analyzed the color-magnitude diagrams (CMDs) of the stellar fields around these Cepheids, in search of anomalously high gradients in the foreground extinction. In all cases, the CMDs are inconsistent with the presence of such nearby foreground clouds, down to angular sizes of $\sim 10^{\prime \prime}$, where the surface density of the disk population becomes too low for drawing such conclusion. That limiting angular scale would correspond to physical sizes of $\lesssim 0.2 \mathrm{pc}$, smaller than a typical Bok globule (Das et al. 2015). The probability for such chance alignment is negligible, and that it affects all 35 objects is virtually zero. Consequently, we rule out the possibility that they are nearby type II Cepheids in the Galactic disk, and classify them as classical Cepheids located inside the bulge volume. Their light curves are presented in Figure 2, while Figure 3 conveys their spatial distribution. Their properties are summarized in Table 1.

By reversing the arguments above, 425 new type II Cepheids are identified in the central bulge (Figures 1(a)-(b), red points), with extinctions consistent with predictions from the VVV reddening map (Gonzalez et al. 2012). If these stars were 

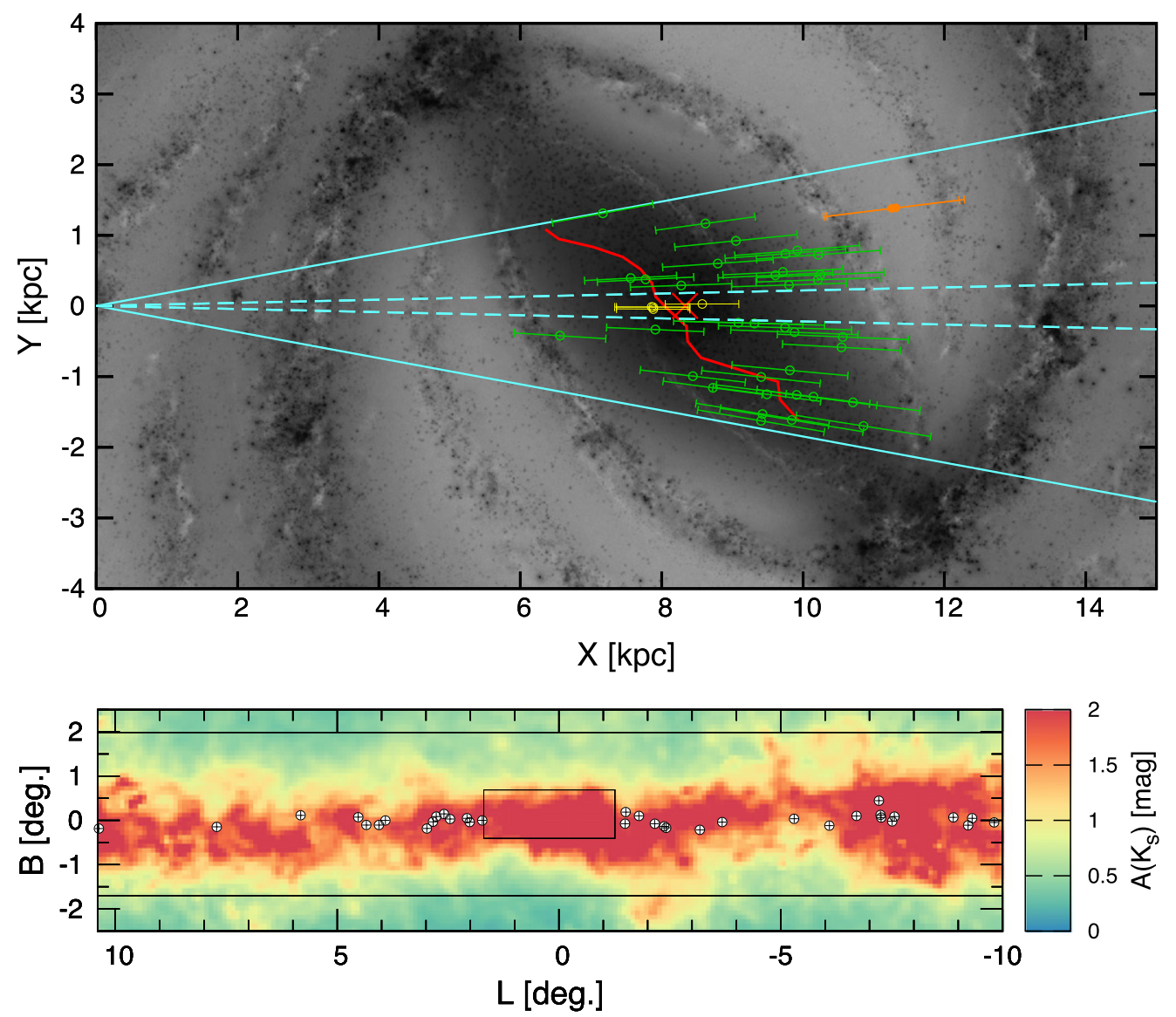

Figure 3. Top panel: positions of the classical Cepheids projected onto the Galactic plane and their errors (green symbols), overlaid on Robert Hurt's illustration of the Milky Way. Orange symbols mark the Twin Cepheids (Dékány et al. 2015) beyond the bulge, yellow symbols represent the classical Cepheids in the nuclear bulge (Matsunaga et al. 2011, 2015). The Galactic bar (Gonzalez et al. 2012) is outlined by a red curve, whereas the red " $x$ " marks the GC (Dékány et al. 2013). The solid and dashed cyan lines denote the longitudinal limits of our study and the direction of the gap in coverage toward the GC, respectively. The bottom panel shows the classical Cepheids" positions in Galactic coordinates, overlaid on the VVV bulge $K_{s}$ extinction map (Gonzalez et al. 2012). Black lines denote the borders of the surveyed area.

classical Cepheids, they would be located far beyond the bulge, and their corresponding extinctions would be inconsistent with such large distances. The detailed study of these objects and the rest of the new classical Cepheids beyond the bulge will be presented in forthcoming papers.

To bolster the classification of the classical and type II Cepheids in our sample, the distributions of their amplitudes and positions were compared. The middle panel of Figure 1 displays the period-amplitude diagram of these objects with previously known classical and type II Cepheids. Although these parameters do not allow us to unambiguously separate the two types, particularly at shorter periods, the locus of the newly discovered classical Cepheid population agrees with that of other classical Cepheids in the Local Group. Furthermore, the right panel of Figure 1 compares the spatial distributions of classical and type II Cepheids identified in this study. While the type II objects are expectedly concentrated toward the GC (Soszyński et al. 2011), the classical Cepheids are rather evenly distributed. The lack of classical Cepheids toward small longitudes, and the slightly offset peak from zero longitude in the density of type II Cepheids, arise from the central gap in the coverage (cf. Figure 3 ).

The sample of 35 new classical Cepheids is incomplete owing to the bright magnitude limit of the VVV Survey. Brighter longer-period Cepheids (Monson et al. 2012) can be saturated in the VVV images in the absence of significant foreground extinction. The asymmetry in the classical Cepheids' observed number density distribution, with more objects at the far side of the bulge (Figure 3), is an observational bias owing to that saturation limit. The typical $K_{s}$ limit where saturation cannot be calibrated out is $\sim 11 \mathrm{mag}$. The faint detection limit toward the central bulge is $K_{s} \sim 15.5 \mathrm{mag}$. These estimates are time-varying, and depend on the seeing, sky transparency, and crowding.

Figure 4 conveys the detection threshold in periodextinction parameter space for classical Cepheids, at various distances. Short-period objects near the $4^{\mathrm{d}}$ lower limit are detected throughout the entire range of observed extinction. However, nearly half of our sample would be overlooked in the absence of significant reddening if they were located at the near side of the bulge. Consequently, the asymmetry in the objects' observed spatial distribution, with more Cepheids further away (Figure 3), is an observational bias owing to the saturation limit of the VVV Survey.

On the other hand, the observed small vertical spread of the classical Cepheids cannot be attributed to a similar observational bias. While long-period classical Cepheids further from the Galactic plane and occupying the bulge would remain undetected by the VVV Survey, more than half our sample would still be detected if they were located at considerably 
Table 1

Catalog of the Classical Cepheids

\begin{tabular}{|c|c|c|c|c|c|c|c|c|c|c|c|c|c|c|}
\hline Obj. & $\begin{array}{c}\text { R.A. } \\
\text { (J2000.0) }\end{array}$ & $\begin{array}{c}\text { Decl. } \\
\text { (J2000.0) }\end{array}$ & $\begin{array}{l}\text { Period } \\
\text { (days) }\end{array}$ & $\begin{array}{c}a_{\text {tot. }} \\
(\mathrm{mag})\end{array}$ & $\begin{array}{c}d_{\mathrm{T}_{\mathrm{T}}} \\
(\mathrm{kpc})\end{array}$ & $\begin{array}{l}A_{K_{s}, \mathrm{~T}_{1}} \\
(\mathrm{mag})\end{array}$ & $\begin{array}{c}d_{\mathrm{T}_{2}} \\
(\mathrm{kpc})\end{array}$ & $\begin{array}{l}A_{K_{s}, \mathrm{~T}_{2}} \\
(\mathrm{mag})\end{array}$ & $\begin{array}{l}A_{K_{s}, \mathrm{~B}} \\
(\mathrm{mag})\end{array}$ & $\begin{array}{c}\sigma A_{K_{s}, \mathrm{RC}} \\
(\mathrm{mag})\end{array}$ & $\begin{array}{c}K_{s} \\
(\mathrm{mag})\end{array}$ & $\begin{array}{c}H-K_{s} \\
(\mathrm{mag})\end{array}$ & $\begin{array}{c}J-K_{s} \\
(\mathrm{mag})\end{array}$ & Note \\
\hline 1 & $17: 22: 23.24$ & $-36: 21: 41.5$ & 7.5685 & 0.22 & $10.99(0.96)$ & 2.07 & $4.40(0.40)$ & 1.94 & 1.44 & 0.21 & $12.01(.02)$ & $1.31(.07)$ & $3.68(.03)$ & $\mathrm{c}$ \\
\hline 2 & $17: 21: 16.05$ & $-36: 43: 25.2$ & 6.3387 & 0.21 & $9.97(1.02)$ & 2.59 & $4.11(0.43)$ & 2.47 & 1.59 & 0.20 & $12.57(.02)$ & $1.62(.10)$ & $4.65(.07)$ & $\ldots$ \\
\hline 3 & $17: 20: 14.62$ & $-37: 11: 16.0$ & 5.0999 & 0.20 & $9.55(0.91)$ & 1.84 & $4.07(0.40)$ & 1.72 & 1.21 & 0.22 & $12.04(.02)$ & $1.16(.08)$ & $3.21(.04)$ & $\ldots$ \\
\hline 4 & $17: 26: 34.72$ & $-35: 16: 24.1$ & 13.4046 & 0.23 & $9.57(0.77)$ & 2.88 & $3.49(0.28)$ & 2.74 & 2.02 & 0.21 & $11.70(.02)$ & $1.81(.05)$ & $5.24(.09)$ & $\ldots$ \\
\hline 5 & $17: 25: 29.70$ & $-34: 45: 45.9$ & 12.3267 & 0.29 & $10.23(0.80)$ & $2.78^{\mathrm{a}}$ & $3.78(0.30)$ & 2.64 & 1.79 & 0.12 & $11.86(.02)$ & $1.75(.04)$ & $5.01(.07)$ & $\mathrm{c}$ \\
\hline 6 & $17: 26: 43.41$ & $-34: 58: 25.6$ & 9.8383 & 0.22 & $9.99(1.15)$ & $4.50^{\mathrm{a}}$ & $3.83(0.44)$ & 4.37 & 1.77 & 0.40 & $13.86(.02)$ & $2.80(.11)$ & $\ldots$ & $\ldots$ \\
\hline 7 & $17: 26: 54.24$ & $-35: 01: 08.2$ & 4.2904 & 0.20 & $10.79(0.95)$ & $3.23^{\mathrm{a}}$ & $4.74(0.44)$ & 3.11 & 1.64 & 0.32 & $13.93(.02)$ & $2.01(.05)$ & $\cdots$ & $\cdots$ \\
\hline 8 & $17: 30: 46.64$ & $-34: 09: 04.4$ & 4.7272 & 0.13 & $9.46(0.84)$ & $3.13^{\mathrm{a}}$ & $4.09(0.38)$ & 3.01 & 1.15 & 0.21 & $13.41(.02)$ & $1.95(.06)$ & $\ldots$ & $\ldots$ \\
\hline 9 & $17: 28: 15.86$ & $-34: 32: 27.2$ & 7.0235 & 0.22 & $8.50(0.75)$ & $3.78^{\mathrm{a}}$ & $3.44(0.31)$ & 3.65 & 1.67 & 0.15 & $13.27(.02)$ & $2.35(.06)$ & $\ldots$ & $\mathrm{d},[1]$ \\
\hline 10 & $17: 38: 42.96$ & $-31: 44: 55.7$ & 11.9663 & 0.28 & $10.56(0.83)$ & 2.81 & $3.92(0.31)$ & 2.67 & 1.61 & 0.22 & $12.01(.03)$ & $1.77(.04)$ & $4.38(.09)$ & $\ldots$ \\
\hline 11 & $17: 36: 44.46$ & $-32: 04: 38.6$ & 8.3220 & 0.17 & $6.58(0.65)$ & $4.40^{\mathrm{a}}$ & $2.59(0.26)$ & 4.27 & 1.31 & 0.33 & $13.09(.03)$ & $2.74(.07)$ & $\ldots$ & $\ldots$ \\
\hline 12 & $17: 40: 41.72$ & $-30: 48: 46.9$ & 10.6634 & 0.22 & $9.89(0.90)$ & 2.91 & $3.74(0.34)$ & 2.78 & 1.73 & 0.27 & $12.13(.04)$ & $1.83(.07)$ & $5.26(.08)$ & $\ldots$ \\
\hline 13 & $17: 40: 25.15$ & $-31: 04: 50.5$ & 4.7004 & 0.16 & $7.92(0.69)$ & 2.19 & $3.42(0.31)$ & 2.07 & 1.79 & 0.22 & $12.10(.04)$ & $1.38(.06)$ & $3.94(.05)$ & $\cdots$ \\
\hline 14 & $17: 42: 20.00$ & $-30: 14: 50.7$ & 23.9729 & 0.42 & $9.09(0.91)$ & $4.16^{\mathrm{a}}$ & $3.02(0.29)$ & 4.01 & 1.51 & 0.25 & $12.05(.04)$ & $2.61(.07)$ & $\ldots$ & $\mathrm{c}$ \\
\hline 15 & $17: 41: 15.13$ & $-30: 07: 17.7$ & 13.3262 & 0.33 & $9.31(1.00)$ & $2.84^{\mathrm{a}}$ & $3.40(0.36)$ & 2.70 & 1.22 & 0.19 & $11.62(.04)$ & $1.79(.10)$ & $5.26(.14)$ & $\mathrm{c}$ \\
\hline 16 & $17: 51: 05.72$ & $-26: 38: 18.3$ & 6.3496 & 0.17 & $9.62(0.81)$ & 2.95 & $3.96(0.34)$ & 2.82 & 1.77 & 0.34 & $12.85(.03)$ & $1.84(.05)$ & $5.26(.07)$ & $\mathrm{c}, \mathrm{d}, \mathrm{e}$ \\
\hline 17 & 17:51:13.77 & $-26: 48: 55.9$ & 12.9488 & 0.25 & $10.25(0.90)$ & $3.11^{\mathrm{a}}$ & $3.76(0.33)$ & 2.96 & 1.41 & 0.27 & $12.13(.03)$ & $1.95(.06)$ & $\ldots$ & $\mathrm{d}$ \\
\hline 18 & $17: 49: 41.42$ & $-27: 27: 14.6$ & 10.4762 & 0.20 & $9.81(0.81)$ & 2.25 & $3.72(0.31)$ & 2.12 & 1.46 & 0.35 & $11.48(.03)$ & $1.43(.06)$ & $4.03(.04)$ & $\cdots$ \\
\hline 19 & $17: 50: 30.49$ & $-27: 13: 46.7$ & 12.6433 & 0.29 & $8.28(0.72)$ & $3.70^{\mathrm{a}}$ & $3.05(0.26)$ & 3.56 & 1.59 & 0.55 & $12.30(.03)$ & $2.32(.05)$ & $\ldots$ & $\cdots$ \\
\hline 20 & $17: 53: 16.07$ & $-26: 28: 26.9$ & 5.9995 & 0.21 & $7.57(0.65)$ & $3.62^{\mathrm{a}}$ & $3.15(0.28)$ & 3.50 & 1.35 & 0.36 & $13.08(.03)$ & $2.25(.05)$ & $\cdots$ & $\cdots$ \\
\hline 21 & $17: 52: 21.66$ & $-26: 31: 19.3$ & 11.9921 & 0.27 & $9.73(0.85)$ & $3.23^{\mathrm{a}}$ & $3.61(0.31)$ & 3.09 & 1.69 & 0.29 & $12.25(.03)$ & $2.02(.06)$ & $5.29(.08)$ & $\mathrm{c}$ \\
\hline 22 & $17: 51: 43.80$ & $-26: 31: 11.3$ & 5.3407 & 0.15 & $7.78(0.68)$ & $3.57^{\mathrm{a}}$ & $3.30(0.30)$ & 3.45 & 1.47 & 0.27 & $13.25(.03)$ & $2.22(.05)$ & .. & $\mathrm{b},[2]$ \\
\hline 23 & $17: 55: 44.68$ & $-25: 00: 30.2$ & 6.7911 & 0.20 & $9.95(0.88)$ & $2.77^{\mathrm{a}}$ & $4.05(0.37)$ & 2.64 & 1.38 & 0.32 & $12.64(.03)$ & $1.73(.07)$ & $5.01(.10)$ & $\ldots$ \\
\hline 24 & $17: 58: 26.68$ & $-23: 52: 08.3$ & 4.5517 & 0.16 & $9.10(0.87)$ & $3.12^{\mathrm{a}}$ & $3.96(0.39)$ & 3.00 & 1.22 & 0.23 & $13.37(.02)$ & $1.94(.07)$ & $5.44(.22)$ & $\ldots$ \\
\hline 25 & $18: 03: 31.12$ & $-22: 21: 14.0$ & 10.9622 & 0.16 & $8.70(0.71)$ & $3.55^{\mathrm{a}}$ & $3.28(0.27)$ & 3.41 & 1.92 & 0.13 & $12.45(.02)$ & $2.22(.04)$ & $\ldots$ & $\mathrm{c}, \mathrm{d}, \mathrm{e}$ \\
\hline 26 & $18: 09: 14.03$ & $-20: 03: 21.4$ & 24.8683 & 0.42 & $7.28(0.72)$ & $5.64^{\mathrm{a}}$ & $2.40(0.23)$ & 5.49 & 0.83 & 0.24 & $13.00(.02)$ & $3.52(.04)$ & $\ldots$ & $\mathrm{c}, \mathrm{d},[3]$ \\
\hline 27 & $17: 22: 10.10$ & $-36: 44: 18.8$ & 14.8719 & 0.33 & $9.55(0.95)$ & $2.77^{\mathrm{a}}$ & $3.43(0.34)$ & 2.63 & 1.47 & 0.28 & $11.44(.02)$ & $1.75(.09)$ & $5.19(.05)$ & b, [4] \\
\hline 28 & $17: 26: 00.10$ & $-35: 15: 15.0$ & 18.2396 & 0.21 & $8.80(0.71)$ & 2.88 & $3.05(0.24)$ & 2.73 & 2.39 & 0.07 & $11.09(.02)$ & $1.82(.04)$ & $5.21(.10)$ & $\ldots$ \\
\hline 29 & $17: 32: 14.07$ & $-33: 23: 59.5$ & 9.9061 & 0.21 & $9.86(0.82)$ & 1.69 & $3.78(0.32)$ & 1.56 & 1.41 & 0.19 & $11.01(.02)$ & $1.08(.07)$ & $2.98(.06)$ & $\ldots$ \\
\hline 30 & $17: 40: 51.51$ & $-30: 24: 53.2$ & 7.9311 & 0.17 & $9.75(0.94)$ & 2.11 & $3.87(0.38)$ & 1.97 & 1.60 & 0.23 & $11.72(.04)$ & $1.33(.09)$ & $3.70(.06)$ & $\ldots$ \\
\hline 31 & $17: 40: 24.58$ & $-31: 01: 32.9$ & 17.3711 & 0.32 & $10.57(0.93)$ & 2.50 & $3.70(0.32)$ & 2.36 & 1.94 & 0.29 & $11.18(.04)$ & $1.59(.06)$ & $4.62(.09)$ & $\ldots$ \\
\hline 32 & $17: 50: 17.54$ & $-27: 08: 13.3$ & 7.0503 & 0.14 & $10.22(0.88)$ & 2.47 & $4.14(0.37)$ & 2.34 & 1.62 & 0.52 & $12.35(.03)$ & $1.55(.06)$ & $4.44(.06)$ & $\ldots$ \\
\hline 33 & $17: 55: 24.20$ & $-25: 30: 22.3$ & 15.4472 & 0.37 & $10.24(0.88)$ & 2.69 & $3.65(0.31)$ & 2.54 & 1.44 & 0.33 & $11.46(.03)$ & $1.70(.06)$ & $5.14(.05)$ & $\ldots$ \\
\hline 34 & $17: 54: 40.25$ & $-25: 34: 39.5$ & 17.1620 & 0.41 & $8.82(0.78)$ & $3.17^{\mathrm{a}}$ & $3.09(0.27)$ & 3.02 & 1.42 & 0.30 & $11.47(.03)$ & $2.00(.06)$ & $5.86(.09)$ & e, [5] \\
\hline 35 & 17:56:01.96 & $-25: 15: 44.9$ & 13.4540 & 0.25 & $9.78(0.85)$ & $2.55^{\mathrm{a}}$ & $3.57(0.31)$ & 2.41 & 1.14 & 0.20 & $11.42(.03)$ & $1.61(.07)$ & $4.59(.04)$ & $\cdots$ \\
\hline
\end{tabular}

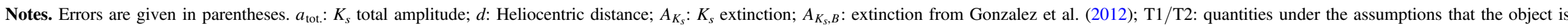

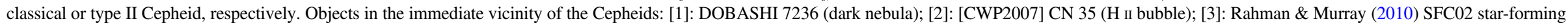
complex; [4]: [CWP2007] CS 96 (H II bubble); [5]: SSTGLMC G003.9104 + 00.0010 (young stellar object).

${ }^{\mathrm{a}} A_{K_{s}, T 1}$ has $>3 \sigma$ positive deviation from $A_{K_{s}, B}$.

${ }^{\mathrm{b}}$ Known ionozed hydrogen bubble close to the Cepheid.

${ }^{c}$ Elevated diffuse mid-infrared emission around the Cepheid at 8- and 24- $\mu \mathrm{m}$ GLIMPSE images.

${ }^{\mathrm{d}}$ Extended bright object close to the Cepheid in 8- and 24- $\mu \mathrm{m}$ GLIMPSE images.

e Young stellar object(s) close to the Cepheid. 


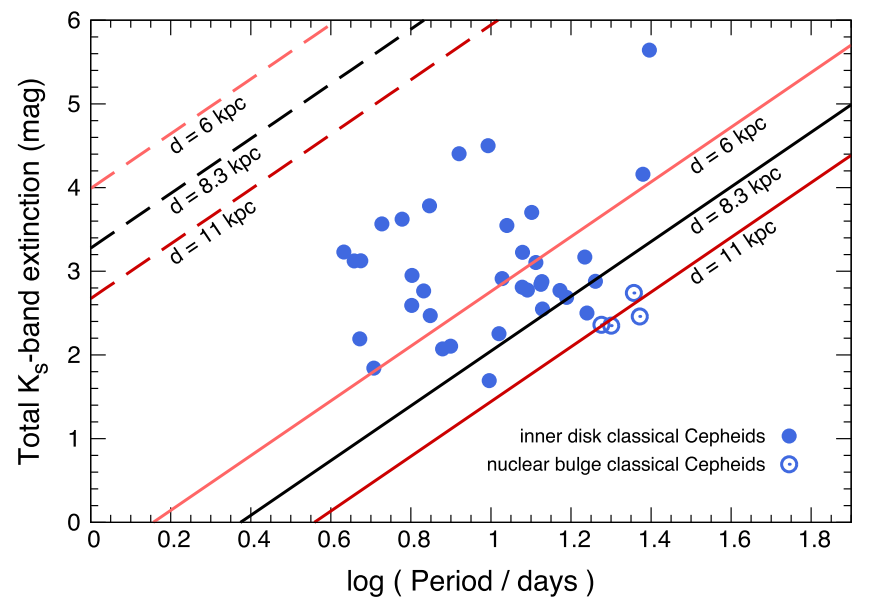

Figure 4. Approximate VVV Survey detection limits, showing the range of pulsation periods and foreground $K_{s}$ extinction where classical Cepheids in the inner Galaxy can be detected. Dashed lines delineate the faint detection limits, whereas solid lines convey saturation limits for Cepheids at three different distances, illustrating the distance to the GC (Dékány et al. 2013) and the corresponding near and far edges of the bulge (Wegg \& Gerhard 2013). Blue points mark the classical Cepheids discovered here, blue circles show the values of classical Cepheids in the nuclear bulge (Matsunaga et al. 2011, 2015).

higher Galactic latitudes. That is apparent when comparing the distributions of Figure 4 and the mean bulge extinction as a function of Galactic latitude in the VVV extinction map (Gonzalez et al. 2012; see also Figure 3, lower panel). Bulge Cepheids exhibiting periods less than 15 days would be detected up to latitudes of $1^{\circ}$ at most longitudes, while those with periods below 8 days would be found at latitudes typically up to $2^{\circ}$.

\section{THE INNER THIN DISK}

The 35 new classical Cepheids inside the bulge volume span across the entire longitudinal range of our study and lie in close proximity to the Galactic mid-plane. The standard deviation of their vertical distances from it is only $22 \mathrm{pc}$, with the farthest Cepheid being only $82 \mathrm{pc}$ above it. This vertical distribution is less than estimates of relatively nearby classical Cepheids (Majaess et al. 2009), whereby the latter exhibit a scale height of $\sim 75 \mathrm{pc}$.

All classical Cepheids discovered are younger than $100 \mathrm{Myr}$, since a Cepheid's pulsation period is closely linked to its age (Bono et al. 2005). The youngest Cepheid observed may be $\sim 25$ million years old (Bono et al. 2005). We cannot exclude the possible presence of even younger and brighter Cepheids, which would be saturated in the VVV Survey. Our objects thus trace an underlying young and thin inner stellar disk along the Galactic plane. Although their census may be incomplete, it is noteworthy that both short- and long-period Cepheids were detected, while in the nuclear bulge only Cepheids with periods close to 20 days are known. The period spread implies that they originate from continuous star formation along the mid-plane in the central Galaxy over the last $\sim 100$ million years.

A limiting factor that hinders a detailed analysis of the spatial distribution of Cepheids in the inner Galaxy is the uncertainty in the wavelength dependence of interstellar extinction, i.e., the properties of interstellar dust particles along the sight-line. However, the bulk of the classical Cepheids in our sample remain within $3 \mathrm{kpc}$ of the GC if we consider different extinction curves (e.g., Cardelli et al. 1989;
Fitzpatrick 1999), even if they have not been observed to be valid toward the inner bulge. For instance, adopting the "standard" Galactic extinction curve (Cardelli et al. 1989) would shift the mean Cepheid distance $\sim 1.5 \mathrm{kpc}$ closer, and only two objects would become further than $3 \mathrm{kpc}$ from the GC (in the near disk). The discovery of a thin star-forming inner disk across the bulge is immune to the effects of potentially anomalous extinction.

Cepheid extinction estimates derived from PL relations were compared to the VVV extinction map (Gonzalez et al. 2012). The comparison is nearly split between values that generally agree, and Cepheids that exhibit significant $(>3 \sigma)$ positive deviations from this map's predictions. These anomalies may originate from localized absorbing material rather than scale height differences between the two populations. Since bulge red clump stars are detected to small angular radii $\left(\sim 10^{\prime \prime}\right)$ around all the Cepheids, most of the material should be distributed inside the bulge's volume and along the sight-lines of objects with extreme extinction. Several of these Cepheids are in the angular vicinity $\left(\lesssim 30^{\prime \prime}\right)$ of ionized hydrogen bubbles, embedded young stellar objects, or extended mid-infrared objects visible in images of the GLIMPSE (Churchwell et al. 2009) surveys (see Table 1).

\section{CONCLUSION}

The presence of numerous classical Cepheids demonstrate that the central Galaxy contains a very young $(<100$ Myr old) stellar population spanning well outside the nuclear bulge. Our results transcend earlier evidence for intermediate-age stars in the bulge volume (e.g., van Loon et al. 2003; Bensby et al. 2013) by exploring low-latitude regions where studies of stellar ages were unobtainable. A hitherto unobserved component of the inner Galaxy was identified, namely a young inner thin disk along the Galactic mid-plane. The confined vertical extent, together with the wide longitudinal breadth of the Cepheids' spatial distribution, suggest that this stellar disk has a smooth transition from both the nuclear bulge and the Galactic thin disk that encompasses the bulge region.

The findings discussed here are in general agreement with recent numerical results concerning the ages of stellar constituents residing in the inner Galaxy. In simulated disk galaxies forming stars from cooling gas inside a dark matter halo, a sizable population of young and metal-rich stars emerged in close proximity to the plane, within a radius of $\sim 2 \mathrm{kpc}$ from the centers. Having a median age of $\sim 1 \mathrm{Gyr}$ and a large age dispersion, these simulated stars show evidence that the presence of young stars in an old bulge can follow naturally from a model galaxy's evolution (Ness et al. 2014). Yet continued investigations are needed to assess whether the Cepheids in the inner disk were born in situ, possibly from star formation stemming from gas inflow toward the GC (e.g., Kormendy \& Kennicutt 2004), or if they originate from further out, either being formed at the bar ends (Phillips 1996) or captured from the outer disk by a growing bar (MartinezValpuesta et al. 2006). Constraining the Cepheids' orbits by kinematical measurements would supply critical information for resolving this point. Another important question is whether the young stars in the inner disk evolve dynamically as old bulge stars once did, and thus will eventually flare into the boxy outer bulge. Such a secular process could supply younger metal-rich stars to the predominantly old outer bulge (Bensby et al. 2013). Given that disk galaxies with small 
bulges such as ours are common, understanding their stellar components' fundamental properties, interactions, and coevolution is key in our quest to understand galaxy evolution as a whole.

We gratefully acknowledge the use of data from the ESO Public Survey program 179.B-2002, taken with the VISTA telescope, and data products from the Cambridge Astronomical Survey Unit. The authors acknowledge the following funding sources: BASAL CATA PFB-06, the Chilean Ministry of Economy's ICM grant IC120009, FIC-R Fund (project 30321072), CONICYT-PCHA (Doctorado Nacional 2014-63140099), CONICYT Anillo ACT 1101, and FONDECYT projects $1141141,1130196,3130552,1120601$, and 1150345.

Facility: ESO:VISTA.

\section{REFERENCES}

Bensby, T., Yee, J. C., Feltzing, S., et al. 2013, A\&A, 549, A147 Bono, G., Marconi, M., Cassisi, S., et al. 2005, ApJ, 621, 966 Brown, T. M., Sahu, K., Anderson, J., et al. 2010, ApJL, 725, L19 Cardelli, J. A., Clayton, G. C., \& Mathis, J. S. 1989, ApJ, 345, 245 Catelan, M., Minniti, D., Lucas, P. W., et al. 2013, arXiv:1310.1996 Catelan, M., \& Smith, H. A. 2015, Pulsating Stars (New York: Wiley-VCH), chap. 7

Churchwell, E., Babler, B. L., Meade, M. R., et al. 2009, PASP, 121, 213

Das, A., Das, H. S., \& Senorita Devi, A. 2015, MNRAS, 452, 389

Dékány, I., Minniti, D., Catelan, M., et al. 2013, ApJL, 776, L19

Dékány, I., Minniti, D., Hajdu, G., et al. 2015, ApJL, 799, L11

Di Matteo, P., Gómez, A., Haywood, M., et al. 2015, A\&A, 577, A1
Fitzpatrick, E. L. 1999, PASP, 111, 63

Gardner, E., Debattista, V. P., Robin, A. C., Vásquez, S., \& Zoccali, M. 2014, MNRAS, 438, 3275

Gonzalez, O. A., Rejkuba, M., Zoccali, M., et al. 2012, A\&A, 543, A13

Irwin, M. J., Lewis, J., Hodgkin, S., et al. 2004, Proc. SPIE, 5493, 411

Kormendy, J., \& Kennicutt, R. C., Jr. 2004, ARA\&A, 42, 603

Launhardt, R., Zylka, R., \& Mezger, P. G. 2002, A\&A, 384, 112

Macri, L. M., Ngeow, C.-C., Kanbur, S. M., Mahzooni, S., \& Smitka, M. T. 2015, AJ, 149, 117

Majaess, D. J., Turner, D. G., \& Lane, D. J. 2009, MNRAS, 398, 263

Martinez-Valpuesta, I., Shlosman, I., \& Heller, C. 2006, ApJ, 637, 214

Matsunaga, N., Feast, M. W., \& Menzies, J. W. 2009, MNRAS, 397, 933

Matsunaga, N., Fukue, K., Yamamoto, R., et al. 2015, ApJ, 799, 46

Matsunaga, N., Fukushi, H., Nakada, Y., et al. 2006, MNRAS, 370, 1979

Matsunaga, N., Kawadu, T., Nishiyama, S., et al. 2011, Natur, 477, 188

McWilliam, A., \& Zoccali, M. 2010, ApJ, 724, 1491

Minniti, D., Lucas, P. W., Emerson, J. P., et al. 2010, NewA, 15, 433

Monson, A. J., Freedman, W. L., Madore, B. F., et al. 2012, ApJ, 759, 146

Monson, A. J., \& Pierce, M. J. 2011, ApJS, 193, 12

Nakada, Y., Onaka, T., Yamamura, I., et al. 1991, Natur, 353, 140

Ness, M., Debattista, V. P., Bensby, T., et al. 2014, ApJL, 787, L19

Ness, M., Freeman, K., Athanassoula, E., et al. 2013, MNRAS, 432, 2092

Nishiyama, S., Tamura, M., Hatano, H., et al. 2009, ApJ, 696, 1407

Ortolani, S., Renzini, A., Gilmozzi, R., et al. 1995, Natur, 377, 701

Persson, S. E., Madore, B. F., Krzemiński, W., et al. 2004, AJ, 128, 2239

Phillips, A. C. 1996, in ASP Conf. Ser. 91, Barred Galaxies, ed. R. Buta,

D. A. Crocker, \& B. G. Elmegreen (San Francisco, CA: ASP), 44

Pietrzyński, G., Graczyk, D., Gieren, W., et al. 2013, Natur, 495, 76

Rahman, M., \& Murray, N. 2010, ApJ, 719, 1104

Serabyn, E., \& Morris, M. 1996, Natur, 382, 602

Soszyński, I., Udalski, A., Pietrukowicz, P., et al. 2011, AcA, 61, 285

van Loon, J. T., Gilmore, G. F., Omont, A., et al. 2003, MNRAS, 338, 857

Wegg, C., \& Gerhard, O. 2013, MNRAS, 435, 1874

Zoccali, M., Gonzalez, O. A., Vásquez, S., et al. 2014, A\&A, 562, A66

Zoccali, M., Renzini, A., Ortolani, S., et al. 2003, A\&A, 399, 931 